\title{
EXTENSIÓN UNIVERSITARIA: FUNCIÓN ORIENTADA AL DESARROLLO REGIONAL
}

Rossana Bastías*

Alejandro Rodríguez** 


\section{RESUMEN}

La extensión universitaria es una de las funciones fundamentales e históricas de la universidad. Este hecho adquiere particular relevancia en regiones, donde constituye un instrumento para el desarrollo en el ámbito cultural globalmente entendido, más allá de las actividades artístico-culturales.

El artículo plantea el rol de las direcciones de extensión como unidades estratégicas para el logro de los objetivos institucionales -en universidades concebidas como entidades interactuantes con el medio social que las alberga-, y la responsabilidad de los académicos en este contexto.

Se exploran también algunos medios propios como el centro de extensión, los sellos editoriales y los programas de colaboración y cooperación. Finalmente, se aborda el tema de la extensión como un factor relevante en el contexto de las comunicaciones corporativas de la institución universitaria.

\section{ABSTRACT}

University outreach activities are one of the main historical functions of universities. This is a particularly relevant fact in outlying regions where it is instrumental to development in the field of culture in comprehensive terms, extending beyond artistic and cultural activities.

The paper discusses the role of outreach departments as strategic units to help achieve institutional goals-at universities conceived as bodies that interact with the social environment of which they form part-and the duties of academicians in this context.

The document also analyzes some roles inherent to universities such as outreach centers, printing houses and programs involving collaboration and cooperation. Finally, the paper addresses the issue of outreach activities as a significant factor in terms of corporate communications by universities. 


\section{EXTENSIÓN UNIVERSITARIA: FUNCIÓN ORIENTADA AL DESARROLLO REGIONAL}

Extensión universitaria: marco conceptual

En la mayoría de las universidades chilenas la extensión es una de sus actividades constitutivas y forma parte de la misión de tales instituciones, junto a la docencia, la investigación y la prestación de servicios. En los textos que la definen se declara que el sentido del quehacer universitario es el desarrollo, conservación y transmisión del conocimiento, la ciencia y el arte en un nivel superior.

En este contexto, la extensión universitaria se entiende como la actividad que da cuenta de la transmisión del saber en una doble dirección: hacia la comunidad externa y hacia la comunidad universitaria, abarcando dos ámbitos de desarrollo: el arte y la cultura, y la extensión académica universitaria

El primero de éstos corresponde a procesos de difusión de la actividad artística y cultural desarrollada al interior de las universidades $y$, a la vez, a la acogida de la actividad creativa de artistas e intelectuales que, desde fuera de la universidad, son integrados por ésta en sus programas de fomento de la actividad cultural. El segundo ámbito, es decir, la extensión académica universitaria, se ha diferenciado del anterior por tratarse de un tipo de actividad que propende a la difusión del conocimiento desarrollado al interior de la universidad, desde un punto de vista disciplinario o como producto de la investigación o la docencia.

Al analizar históricamente el concepto de extensión en su sentido más amplio, es decir, aquel que abarca tanto la actividad de difusión del arte y la cultura como la de difusión de lo académico propiamente 
tal, podemos distinguir un paso que va desde una mirada asistencialista, en la que se trata de traspasar un conocimiento cultivado a una comunidad que no lo posee o no tiene acceso a éste, pasando luego, en la década del 60, a formar parte de uno de los instrumentos de promoción del cambio social, hasta una actividad orientada a la generación de recursos y a la difusión del arte y la ciencia ${ }^{1}$.

Por otra parte, hay que atender a la evolución de la idea de universidad, cuya dinámica nos lleva desde una institución que se autodefine en función de sí misma, hasta la actual, integrada e interactuante con el medio social, económico y cultural, y de la cual se demanda, socialmente, una cada vez mayor vinculación con el desarrollo de la ciencia, la tecnología, el arte y la cultura en general.

Este modelo de universidad, integrada e interactuante con el medio, es el contexto en que la extensión se constituye en una actividad propicia para generar las relaciones institucionales y corporativas necesarias para contribuir al cumplimiento de una misión que va mucho más allá de la enseñanza de unas profesiones tradicionales. La universidad contemporánea es una red de interacciones, conversaciones y vinculaciones, que integran conocimientos, metodologías, investigaciones, reflexiones y acciones diversas, y que contribuyen al desarrollo de la comunidad en que se encuentran insertas.

En Chile, en particular, la existencia desde 1981 de las denominadas universidades regionales, fundadas a partir de las sedes regionales de las Universidad de Chile y de la Universidad Técnica del Estado, junto a la presencia de otras instituciones de carácter tradicional y privado, como las universidades católicas u otras instituciones como la Universidad Santa María o de Concepción, han generado un importante impacto en el desarrollo de sus respectivas regiones no sólo en la formación de profesionales, sino en su interrelación con el medio a través de proyectos, convenios, investigaciones, actividades artísticas y culturales, entre otras acciones,

1 Sicard, Andrés, "Significado y sentido de la extensión", Actas del III Encuentro Nacional de Extensión Universitaria: Diálogo de Saberes, Corporación Universitaria Autónoma de Occidente, Cali, octubre 22 y 23 de 2003, disponible en http://www.ascun.org.co/foro/eeu/eeu.htm 
que demuestran que la universidad hoy se integra con su comunidad en diversas dimensiones del quehacer humano.

En este sentido, concebimos la extensión como una actividad estratégica de las universidades que permite su integración con el medio social, contribuyendo a la generación de relaciones cada vez más complejas, posibilitando que las temáticas ciudadanas penetren a la universidad y que el quehacer universitario sea valorado por ésta. La extensión es, por lo tanto, una actividad de comunicación que fluye en ambos sentidos, creando una instancia de diálogo que enriquece a sus interlocutores. Las direcciones de extensión deberían, por lo tanto, ser parte de la alta dirección de las universidades, constituyéndose en una instancia para la definición de políticas de interacción con la sociedad, que sea capaz de articular cada una de las instituciones de educación superior de acuerdo a sus propias realidades. Se trata de generar las coordinaciones necesarias para articular las redes internas capaces de responder a las demandas generadas desde el ámbito social. De este modo, la misión y los objetivos de la extensión se tornan coherentes con los de la institución en general y se articulan convenientemente con el ámbito público y privado.

La extensión académica universitaria: re-imaginar la región

En el contexto antes descrito, la universidad, y particularmente la universidad regional, deben asumir el desafío de pensar, imaginar y desarrollar la región.

Es en la universidad donde se encuentran la inteligencia y la energía de los jóvenes junto al conocimiento de los académicos. La universidad tradicional es una estructura de carácter jerarquizado, en la que los académicos mayores dirigen el trabajo de los más jóvenes y las comunidades locales establecen los vínculos necesarios con el sector público y el sector privado, para detectar, conocer y desarrollar soluciones a los problemas regionales.

Por ello, la extensión académica, entendida como la actividad de difusión del quehacer de académicos y estudiantes en el marco de las disciplinas que se cultivan en la universidad, es un quehacer integrador, fundado en la vinculación y la gestión de relaciones internas y externas 
de la institución, que colabora en la estructuración de redes y equipos multidisciplinarios orientados al desarrollo de proyectos y a la creación de instancias de reflexión y diseño del futuro.

Un objetivo de esta naturaleza demanda un perfil académico con capacidad de gestión, que cultive las relaciones institucionales y represente a la universidad en todas sus actuaciones públicas; es decir, un académico que trascienda su función docente y abra sus sentidos y su capacidad intelectual a los enormes desafíos que la globalización impone al desarrollo regional y local. A nuestro juicio, hoy los académicos universitarios deberían tener presente, en todo momento, su rol de extensión como una más de las tareas a las que se abocan en su quehacer cotidiano.

Si las universidades son, por naturaleza, espacios para la reflexión y el estudio, entonces conforman el mejor lugar para generar las condiciones en las cuales dicha reflexión tenga lugar, y se estrechen los lazos entre la visión académica y la realidad pública y privada.

La universidad, en conjugación con autoridades y organismos de fomento -considerando la diversidad de las disciplinas que se cultivan en sus aulas, la capacidad profesional de sus docentes, sus instalaciones, laboratorios y bibliotecas- puede constituirse en un espacio de enorme potencial para generar proyectos, contribuyendo al desarrollo de la imagen objetivo que se requiere construir en las regiones para lograr visiones de futuro capaces de movilizar los esfuerzos del sector público y privado, generando sinergias integradoras que potencien el desarrollo económico y cultural. El gran desafío es pensar la región desde la región y, para ello, se hace necesaria la integración transversal de la universidad, las organizaciones intermedias de la sociedad civil, el sector público y las organizaciones intermedias del sector privado. Este rol de integración, coordinación, reflexión continua y articulada transversalmente debiera ser asumido por la extensión universitaria como parte de la misión que les corresponde a las instituciones universitarias regionales.

Pensar la región desde la perspectiva cultural general, económica y política, identificando las debilidades, fortalezas, oportunidades y amenazas, constituye hoy un deber ineludible de la extensión universitaria, mediante la organización de jornadas de reflexión y la creación 
de redes que involucren a todos los actores. Ya no es posible considerar a la universidad como un ente aislado de su entorno, desvinculado de los problemas que atañen y aquejan a la población de su región, y como un lugar en el que se aprende un conocimiento abstracto y sin aplicación. Esto, por otra parte, implica que la sociedad, las autoridades, el sector empresarial y la sociedad civil integren a sus universidades a la definición de sus prioridades de desarrollo y, a la vez, que las universidades acepten el desafío de participar de tales definiciones, cambiando su cultura de claustros desligados de la vida real.

Ciclo de causalidad de la extensión

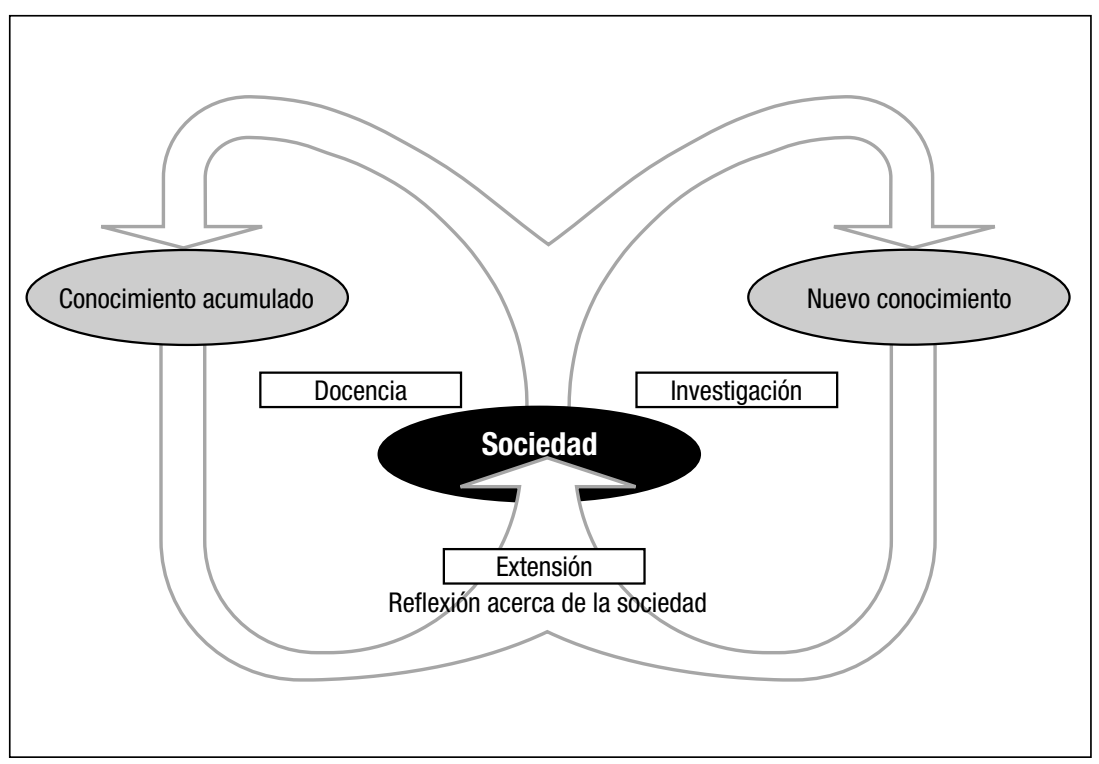

En el ámbito de la docencia, la labor de la universidad es la formación de personas capaces de aprender por sí mismas, de ciudadanos críticos, sensibles, tolerantes, con reconocimiento de la diversidad y pluralistas. Para ello se requiere que las aulas se impregnen no sólo de las clases dictadas por los profesores; en una sociedad del conocimiento, también la educación de una mirada amplia y culta acerca de la sociedad resulta imprescindible para la formación de ciudadanos que valoren la democracia y la participación colectiva, 
ciudadanos con un marco referencial que vaya más allá de sus particulares disciplinas y áreas de estudio específicos. A nuestro juicio, la extensión universitaria, entendida como una función capaz de captar el sentir ciudadano y las preocupaciones de la sociedad, por su propia transversalidad, constituye la actividad pertinente para estos fines.

En síntesis, la función de la extensión universitaria y, en particular, el rol de la extensión académica deben centrarse en la creación e implementación de instancias de reflexión que hagan posible la interacción entre los actores sociales, a fin de pensar e imaginar continuamente la región desde su propio seno, adelantando los desafíos y focalizando las metas del desarrollo humano.

Para que ello sea posible, se requiere una valoración social de la actividad extensional y una valoración institucional de esta función universitaria que, en la mayoría de los casos, se encuentra muy por debajo de la docencia y de la investigación. Si como país estamos apostando al desarrollo regional, entonces la articulación y constitución de instrumentos e instancias de relación entre las universidades, los gobiernos regionales y las organizaciones de la sociedad civil permiten soñar con una valoración mayor de la extensión como la actividad que, en la estructura organizacional de las universidades, se ocupe de estos temas. Por ello, los esfuerzos desplegados por el Programa Universidades y Gobiernos Regionales son un buen ejemplo de lo que se puede lograr. Falta aún que la actividad de extensión sea convenientemente valorada en el sistema de acreditación al que se ha convocado a las universidades, generando indicadores de desempeño que permitan medir la eficiencia y eficacia con las que la extensión contribuye al logro de los objetivos institucionales y regionales.

La extensión cultural como factor de desarrollo: educar la sensibilidad

Un análisis realizado a partir de las páginas web de las instituciones universitarias del país permite afirmar que la mayoría de las universidades desarrolla actividades en el campo de la extensión cultural en su acepción restringida: la que relaciona esta actividad con las expresiones artísticas.

Al decir esto, no estamos desvalorizando este tipo de acción. La extensión artística constituye una actividad relevante desde el punto 
de vista de la ampliación de los espacios para la apreciación estética, la aceptación de la diversidad y el cultivo de la tolerancia, teniendo en cuenta que los artistas, en general, conforman un grupo de personas altamente contestatarias, que buscan provocar en el espectador una reflexión acerca de su propuesta ética, estética y, en muchos casos, política.

En este sentido la universidad se torna un espacio para exhibir estas propuestas, contribuyendo, de este modo, a la creación de instancias para que se desarrollen los valores antes señalados; valores que, como sociedad democrática, debemos ser capaces de cultivar ampliamente.

Al encontrarse inmersas en una realidad alejada culturalmente del centro político y económico del país, las regiones no tienen acceso directo a las manifestaciones más desarrolladas del arte, reduciendo de este modo la mirada que construyen sobre sí mismas y generando un círculo vicioso que no conduce a la construcción de visiones de desarrollo. En este contexto, los únicos lugares para que esta posibilidad se desarrolle están dados por los espacios públicos de carácter universitario, los que, en muchas ocasiones, sobreponiéndose tanto a la crítica interna como externa, hacen posible que tales sensibilidades se expresen, aunque contravengan las concepciones culturales dominantes.

Bien gestionadas, las actividades de extensión artística, especialmente si se vinculan con actividades de extensión académica que den pie a la reflexión y al análisis crítico, permiten ampliar los horizontes interpretativos y educar la sensibilidad estética de nuestras comunidades, la mayor parte de las veces sometida a los discursos de los medios de comunicación de masas que sobreponen los intereses comerciales a los culturales, reduciendo las posibilidades de hacer crecer la capacidad de expresión de la sociedad y generando una aturdidora uniformidad en el discurso público y privado.

\section{El centro de extensión cultural}

Si la extensión artística y cultural, en su acepción amplia, debería estar orientada al logro de objetivos de articulación de la participación, la reflexión colectiva y el fomento de instancias de creación 
artística -para la construcción de una sociedad diversa, tolerante, sensible, conformada por personas capaces de diseñar realidades nuevas a partir de sus marcos referenciales enriquecidos y dinámicos-, las universidades, y las diversas instancias gubernamentales y civiles que coinciden con tales objetivos, deberían desarrollar los instrumentos que hicieran posible crear las condiciones para lograr tales efectos.

A nuestro juicio, uno de tales instrumentos, especialmente en el caso de las regiones, es la constitución de centros de extensión en las principales ciudades de nuestro país, en los que se concentre -en forma paralela, continua y estructurada- la actividad extensional en sus acepciones ya mencionadas.

Como sabemos, uno de los aspectos que marcan con mayor fuerza una cierta inequidad en la distribución de los bienes culturales en el país es la escasez de recursos financieros y de infraestructura. A pesar de lo mucho que se ha avanzado en estas materias desde 1990, con la constitución del Fondo Nacional de Desarrollo Cultural y de las Artes (Fondart), aún persiste una falta de espacios técnicamente equipados para recibir exposiciones, obras musicales y de teatro, ballet y ópera de envergadura, concentrándose tales recursos principalmente en el centro del país, con algunas pocas excepciones.

Los centros de extensión, articulados a partir de un equipo de recursos humanos formados en la gestión de la cultura, las comunicaciones visuales y periodísticas, hacen posible la creación de lugares perfectamente identificados en la trama urbana, en los que se proyecta el quehacer cultural en dimensiones mayores. Espacios para la creación, la reflexión y difusión del saber artístico, científico y humanístico, que permiten, a quienes lo visitan, acceder a una ventana hacia la cultura local, nacional y universal, articulada con los objetivos de desarrollo de la región.

Se suma a lo anterior la existencia de sellos editoriales, radios locales y, en algunos casos, canales de televisión que no han sucumbido al mercado y se mantienen en el ámbito universitario y cultural que los vio nacer.

Todos ellos conforman un sistema capaz de generar un impacto de gran envergadura, si su gestión se estructura en función de una 
perspectiva de mediano y largo plazo y se define con una mirada que vaya más allá de la actividad propia de cada universidad.

La extensión cultural: un espacio para la colaboración.

La extensión debería ser entendida como una actividad eminentemente colaborativa a la que concurran con sus capacidades, puntos de vista y recursos todas las instituciones, personas y entidades públicas y privadas interesadas en el desarrollo de los objetivos antes señalados. Si bien en aquellas ciudades en las que existen sedes de diversas universidades se debe dar una legítima competencia por la captación de alumnos y por el posicionamiento de sus respectivas imágenes corporativas, esta competencia debería reducirse al ámbito de las carreras que se imparten, reservando los planos de la investigación y, principalmente, la extensión para la construcción de una cultura de la colaboración e integración, en beneficio de un objetivo de mayor alcance cual es el desarrollo cultural de las regiones.

En este sentido, el caso de la Comisión de Extensión del Consejo de Rectores de Valparaíso se puede considerar como un buen ejemplo. En efecto, por cerca de treinta años ha sido la instancia de articulación de actividades coordinadas entre las universidades tradicionales de esta ciudad. Desde 1990 constituyó una Comisión de Extensión que es presidida por el Director de Extensión de la Universidad, cuyo rector preside a su vez el Consejo con una secuencia rotatoria de un año de duración. Esta estructura ha permitido desarrollar una multiplicidad de actividades, potenciando las capacidades de cada una de las universidades participantes: ciclos de conciertos, exposiciones, giras musicales, teatro, cine, conferencias y encuentros académicos de diversa índole. Ello facilita, asimismo, la relación con entidades públicas y concentra la obtención de recursos financieros desde el sector privado, que se ve beneficiado por las leyes de exención tributaria para donaciones de carácter cultural.

Extensión cultural y editorial

Otro poderoso instrumento de la extensión universitaria, en el sentido en el que la hemos definido en el contexto de este artículo, es la gestión de un sello editorial. 
Por su misma función, un sello editorial es un órgano de difusión del quehacer académico interno surgido de la actividad investigadora y docente, como también un elemento catalizador de la actividad intelectual y artística de la región. Múltiples son los ejemplos de cómo una gestión editorial inteligente y asociada a los objetivos de desarrollo regional puede ser multiplicadora de la actividad creativa y generadora de otras tantas actividades de reflexión asociadas. Un sello editorial es capaz de recoger y comunicar los elementos de la identidad regional y local, potenciando la necesaria mirada de ensimismamiento que deben realizar las regiones para definir sus propios perfiles y objetivos de desarrollo. En este sentido, el valor de la palabra escrita y su aliado el libro es aún el mejor invento humano para mantener y poner de relieve la memoria y, por ende, la identidad a partir de lo cual se pueden proponer visiones de desarrollo. La persistencia de la palabra hecha libro es, hasta ahora, insuperable. Por esto, un sello editorial puede constituirse, a través de sus publicaciones, en un poderoso instrumento a la hora de influir en la opinión y en la definición de los caminos del desarrollo regional.

Extensión y comunicaciones. Una sinergia que incide en la imagen corporativa

Si entendemos la comunicación como "oportunidad de encuentro con el otro", y la extensión como un proceso de comunicación, es clave para el desarrollo de la extensión cultural universitaria cómo las universidades se plantean y articulan programas de extensión que den posibilidad de interacción en el ámbito interno y externo de la organización universitaria. Se trata de generar condiciones de visibilidad, que permitan la coordinación y la cooperación con otras instancias sociales de desarrollo local y el crecimiento de la universidad y sus actores: los directivos, académicos, administrativos y estudiantes.

La universidad, como institución de educación superior, debe contar en su estructura organizacional con un órgano de comunicaciones que se coordine con la extensión universitaria, que permita generar hacia su interior una suerte de sentimiento de pertenencia y proyectar en el ámbito externo una visualización reconocible de la organización. 
Históricamente, la universidad, como organización social, ha tenido como misión el desarrollo de la docencia, la investigación y la extensión académica y cultural; estas últimas funciones se han visto enriquecidas por los conceptos renovados de la comunicación corporativa. Ello, a su vez, ha implicado un nuevo desarrollo en el plano de los contenidos de comunicación y en el de las nuevas tecnologías de la información, que han posibilitado a las universidades estar presente en los nuevos medios de comunicación desplegando un discurso propio, ya no mediado por las empresas de los medios masivos de comunicación como son las radioemisoras y las cadenas periodísticas del país.

Las universidades, como organizaciones humanas y a través de sus direcciones de extensión, han implementado procesos comunicacionales como recurso para integrarse a la sociedad, potenciando su desarrollo. Ello ha hecho posible el reconocimiento de las comunicaciones como un valor estratégico para sobrevivir en un mundo altamente competitivo y cambiante, en el cual la creación y gestión de imagen resulta relevante.

Como organizaciones que producen servicios en el ámbito del conocimiento y la cultura, es decir intangibles, las universidades necesitan cada vez más la implementación de estrategias de comunicación corporativa, como "función sustancial asociada a la cultura, a la identidad, a la imagen y a la comunicación de la organización" 2 .

La construcción de la identidad de las universidades se desarrolla con su forma de ser, propio de esta tipología de organización, que es una organización compleja desde el punto de vista del comportamiento de sus actores y su identidad. La identidad corporativa de las universidades se obtiene de la articulación de sus signos visuales (marca), de su infraestructura (edificios) y de la conducta global de su colectivo interno (directivos, académicos, administrativos y estudiantes).

2 Costa, Joan, Comunicación corporativa y revolución de los servicios, Editorial de las Ciencias Sociales, Madrid, 1995. 
Mantener la identidad de su universidad es un deber de los colectivos internos, que deben contribuir a la construcción de la imagen; también es tarea de las direcciones de comunicación, que se constituyen así en factores estratégicos en el proceso de gestionar y comunicar el quehacer de esta organización.

Las universidades tradicionales son organizaciones que han debido ir adaptando su estructura al devenir de los cambios del entorno social y tecnológico. A pesar de ello, deben mantener su espíritu, entendido como la cultura e identidad del ser universidad que en estos tiempos es sinónimo de educación superior. Frente a este desafío, el filósofo Humberto Giannini nos dice: "Y esto va mucho más allá de un problema definitorio: se trata de satisfacer, por una parte, las exigencias del nuevo dinamismo y elasticidad del mercado profesional, $\mathrm{y}$, por otra, de asegurar desde una perspectiva más responsable y reflexiva que, al menos, este mercado no pierda de vista los fundamentos teóricos y las razones sociales y éticas que deben regular su vocación centrífuga"3.

Por ello, podemos concluir que todas las acciones de investigación y de extensión cultural y académica que realizan los colectivos internos de las universidades son significantes y están sometidas a la interpretación de la sociedad en su conjunto, y será ésta la que construya una buena o mala imagen a partir de los procesos comunicativos de acción de la organización-universidad y la reacción de la sociedad-individuos.

3 Giannini, Humberto, "Universidad transocial y globalización cultural", en: División de Educación Superior, El Impacto de la Globalización en la Educación Superior, Mineduc, Santiago de Chile, 1998. 